study, would not migrate as anions in an electrical field, and in any phenol extraction from cellular materials would partition in the organic (not the aqueous) layer, and so on. However, they could still form Watson-Crick base pairs and thus might genetically respond normally. Conceivably, the modification could even be used to anchor the nucleic acid to the prion protein, in the holoprion model. Like Weissmann's model, this suggestion could account "for most if not all experimental observations, does not involve unknown mechanisms and makes testable predictions". But, as he states for his model, "it may also be wrong". To the best of my knowledge, no one has looked for a 'camouflaged' DNA as a factor in spongiform encephalopathies.

BEVERLY E. GRIFFIN

Royal Postgraduate Medical School, London W12 ONN, UK

\section{Out on a limb}

SIR - In 1987, a paper ${ }^{1}$ and accompanying News and Views article ${ }^{2}$ were published in Nature in which it was suggested that retinoic acid is a morphogen. Since then there have been many reports purporting to confirm this claim. A widely used textbook in cell biology ${ }^{3}$, for example, states that with regard to determination of the skeletal pattern in the vertebrate limb, "it is very likely that retinoic acid is the natural morphogen".

Previous work had shown that ectopic grafts of an extra zone of polarizing activity (ZPA), a portion of the normal limb bud ${ }^{4}$, as well as resin implants containing retinoic acid $^{5}$, could induce skeletal duplications during limb development. The authors of the 1987 paper ${ }^{1}$ showed that ZPA itself is indeed relatively rich in retinoic acid. The claim that retinoic acid is an endogenous limb morphogen could have been tempered, however, by acknowledgement of the long-established fact that the endogenous ZPA is entirely dispensable for normal skeletal development during the period of limb pattern formation ${ }^{6}$. And the implausibility of the notion that a gradient of a single substance, such as retinoic acid, could determine where skeletal elements would form, and their separate identities as well, might also have been considered. When readers of Nature learned earlier this year ${ }^{7,8}$ that retinoic acid is almost certainly not a morphogen in the developing limb, those outside the field must have been more than a little surprised.

This episode would be of only historical interest if the same phenomenon were not cropping up again with regard to the new 'master regulatory molecules' of the day, the homeobox gene products. Such molecules are clearly of importance in establishing and reinforcing segmental prepatterns and segment identities in insects. But their roles in pattern formation in nonsegmented organisms such as the nematode Caenorhabditis elegans are a matter of speculation ${ }^{9}$, and nothing whatever is known about their causa role in limb skeletal pattern formation. Nonetheless, articles are beginning to appear on the distributions of homeobox gene products in the vertebrate limb (none of which, in fact, actually resembles the skeletal pattern) with claims like "Hox-4 genes probably encode positional information" 10 . I believe that it would be preferable to apply the standard that proposed interpretations of developmental pattern formation should attempt to account for why a pattern looks like it does, and not like something else.

\section{Department of Cell Biology and} Anatomy,

New York Medical College, Valhalla, New York 10595, USA

SLACK REPLIES - How can we know whether a molecule is really acting as a morphogen? Like many others I have spent some time thinking about this and have published a set of possible criteria $^{11}$. To simplify these somewhat: the substance should have the expected biological activity; it should be present at the right time, place and concentration; and appropriate inhibition should inhibit the effect in vivo.

Back in 1987, it seemed to me sufficient that retinoic acid had the right effects and had the predicted distribution in vivo. Retinoic acid enters and leaves cells very easily, so a steady-state concentration difference guarantees diffusion from regions of high to regions of low concentration. Since then, other active endogenous retinoids have been discovered ${ }^{12}$, it is clear that there are several retinoic acid receptors, and the situation is obviously more complex than it appeared at first sight. A satisfactory inhibition experiment has yet to be devised. So perhaps my News and Views piece $^{2}$ should have been entitled "We think we have a molecule that looks more like a morphogen than any before", but I am not sure that the editors would have liked that as much.

As to Newman's second point, I do not agree. Experimental embryology teaches us that the causal mechanisms in regional specification do not 'look like' the final pattern. The model of a morphogen gradient with threshold responses was formulated to account for the embryological data ${ }^{13}$. Now we have some candidate morphogens, the retinoids, and some candidate primary response elements, the Hox-4 genes, and this seems like progress to me. Newman is right to emphasize that we do not understand, even for the primary body plan of Drosophila, the links between the 'serial threshold' arrangement of Hox gene expression and the later visible pattern of cell differentiation. But you can't solve everything at once, and recent progress in developmental biology has been quite spectacular enough.

J. M. W. SLACK

ICRF Developmental Biology Unit, Department of Zoology,

University of Oxford,

Oxford OX1 3PS, UK

1. Thaller, C. \& Eichele, G. Nature 327, 625-628 (1987)

2. Slack, J.M.W. Nature 327, 553-554 (1987).

3. Alberts, B.M. et al. Molecular Biology of the Cell 2nd edn p.917 (Garland, New York, 1989).

4. Saunders, J.W. Ir in Vertebrate Limb and Somite Morphogenesis (eds Ede, D.A., Hinchliffe, J.R. \& Balls, M. 1-24 (Cambridge Univ Press, 1977).

5. Tickle, C., Lee, J. \& Eichele, G. Devl Biol $109,82-95$ (1985)

6. Fallon, J.F. \& Crosby, G.M. J. exp. Zool. 193, 449-455 (1975)

7. Wanek, N., Gardiner, D.M., Muneoka, K. \& Bryant, S.V. Nature 350, 81-83 (1991)

8. Noji, S. et al. Nature 350, 83-86 (1991)

9. Kenyon, C. \& Wang, B. Science 253, 516-517 (1991)

10. Izpisúa-Belmonte, J.-C. et al. Nature 350, 585-589 (1991)

11. Slack, J. M. W. \& Isaacs, H. V. Development 105 147-154 (1989)

12. Thaller, C. \& Eichele, G. Nature 345, 815-819 (1990) 13. Tickle, C., Summerbell, D. \& Wolpert, L. Nature 254 199-202 (1975)

\section{Lead correction}

SIR - S. Weiner and G. Goodman (Nature 352, 385; 1991) correctly recommend that, because of inaccuracies, two figures I prepared relating indices of children's lead exposure to their IQ scores should not serve as a basis for policy decisions on acceptable lead levels. They neglected to mention, however, that the inaccuracies have been corrected. The revised versions of these figures also identify the nature and source of the data depicted from each study. I became aware of the need to revise the figures in March, told Weiner this in April and announced it at a meeting on 22-23 April of the US Centers for Disease Control Advisory Committee on childhood lead poisoning. Any reader who wishes copies of the revised figures is invited to contact me.

I apologize to those who may have been misled. But it is important to point out that the basic message conveyed by the figures remains unchanged. The purpose of displaying the data in this manner was to assess the qualitative similarity in the direction and magnitude of the dose-effect relationships reported in various studies, regardless of whether the relationship was significant at $P<0.05$. The quantitative similarity of the findings from many of the studies depicted has been confirmed by meta-analysis. (H. Needleman and C. Gatsonis J. Am. med. Ass. 263, 673; 1990).

DAVID BELLINGER

Neuroepidemiology Unit,

Gardner House, Children's Hospital

Boston, Massachusetts 02115, USA

NATURE · VOL $354 \cdot 7$ NOVEMBER 1991 